means that a study of one part of the subject is enriched by a knowledge of the rest. The primary theme that he has chosen is that of elementary excitations with a secondary theme of the dual descriptions of extended and localised states; and this has particular relevance to imperfect systems. The effects of uniform electric and magnetic fields have been included and most of the experiments which are discussed involve either absorption of light or simple transport properties.

The book uses the compact notation of second quantisation but calculations are performed using time-independent perturbation theory. This has the advantage that the new physical ideas

\section{Psychopharmacology handbook}

Handbook of Psychopharmacology. Edited by L. L. Iversen, S. D. Iversen and S. H. Snyder. Vol. 7 : Principles of Behavioral Pharmacology. Pp. 453. \$41.40. Vol. 8: Drugs, Neurotransmitters, and Behavior. Pp. 590. \$47.40. Vol. 9: Chemical Pathways in the Brain. Pp. 410. \$35.40. (Plenum: New York and London, 1978.)

WITH these three volumes the IversenSnyder Handbook moves from the field of basic neuropharmacology into behavioural pharmacology. A total of 30 chapters contributed by 47 authors encompasses a range of subjects from the analysis of operant schedules (Dews and de Weese) to the localisation of gabaminergic neurones (Fonnum and Storm-Mathisen). The contributions are of a high standard and many are highly readable, but it is inevitable in an undertaking of this size that their relationship one to another is often uncertain. Thus, each author attempts to produce a major review of his topic, but is bound to cover material which is also included elsewhere either in these or earlier volumes. Handbooks (and Handbücher) have always suffered from this problem, but of the genre the Iversen-Snyder production has much to commend it. In few other places can one find so much information relevant to the discipline.

Psychopharmacology is nothing if not cross-disciplinary. This should be most evident where two well established disciplines-for example, Psychology and Pharmacology-meet, as they do in volume 7 (Principles of Behavioral Pharmacology). It is interesting to see the extent to which one field has influenced the other. In some cases the impact seems to have been small In the Dews and De Weese review referred to above drugs appear as are not obscured by unfamiliar formalism. There is a useful list of problems and topics for discussion to help the student assimilate the material. A further strength of the book is its excellent bibliography which includes a large number of review articles as well as books and original papers.

I find myself convinced that there is a place for a book of this length at this level and would recommend Professor Madelung's book, particularly to new graduate students.

Gillian Gehring

G. A. Gehring is the Physics Tutor at St Hugh's College, and a Lecturer in the Department of Theoretical Physics at the University of Oxford, UK.

another environmental variable to be accommodated by the same Skinnerian framework that describes other aspects of behaviour. The fact that such agents may be acting upon specific chemical mechanisms within the brain, and that these mechanisms may be regulating specific aspects of behaviour, is regarded as irrelevant to the description of drug action. The purely inductive approach seems, in this case, to act as a defence against asking some obvious, if difficult, questions. From a different psychological viewpoint Milner demonstrates a similar insularity. In giving a useful introductory survey to theories of motivation and learning which would be at home in a textbook of psychology, followed by some neural models, Milner implies that all this may be relevant to recent work on the physiology of central monoamine systems. Yet when it comes to making specific predictions from his own theory, or assessing the progress of other theories of the functions of monoamine neurones, Milner is reticent. It is at this point that the discipline of psychopharmacology (if there is one) gets interesting.

By contrast the work reviewed by Marshall and Teitelbaum (Neuropsychology of Motivated Behaviours) reveals the contribution which a rigorous application of the techniques of neuropsychology to preparations derived from advances in neuropharmacology (6-hydroxy-dopamine induced lesions of dopamine neurones) can make to our understanding of the mechanisms of behaviour. The theoretical implications of this work are considerable, though not developed at length by these authors, and give support to the view that neuroscience is in some respects leaving psychology behind. Other chapters in this volume cover major technical questions (for example, Robbins on motor activity, Mackintosh and others on ethological contributions and methods). Kumar has contributed a succinct but wide- ranging review of animal models of relevance to psychiatry, attempting to synthesise psychodynamic (for example, object loss), ethological, operant (for example, learned helplessness) and neuropharmacological, hypotheses. New dimensions to psychopharmacology are reviewed by Broadhurst (genetics) and Mabry and Campbell (development).

Many issues in volume 7 re-appear in volume 8 (Drugs, Neurotransmitters and Behavior), sometimes in a more controversial form. Here major motivated behaviours (eating, drinking, sleep) or motivational constructs (reward, punishment, fear, frustration) are foci for a discussion of pharmacological, behavioural and often many other aspects. Sometimes the relevance for psychopharmacology is obscure. For example, in a somewhat partisan account of the anatomy of selfstimulation, Routtenberg and SantosAnderson make a virtue of discounting the neuropharmacological interest of the reward phenomenon. To this reviewer it remains an interesting and viable hypothesis that all reward

\section{Applied group theory}

Induced Representations in Crystals and Molecules: Point, Space and Nonrigid Molecule Groups. By S. L. Altmann. Pp. 369. (Academic: London, New York and San Francisco, 1978.) $£ 18$; $\$ 32.25$. Review by H. C. LonguetHiggins, Nature, 274, 403 (1978).

Dr Altmann has drawn the Editor's attention to certain sentences in Professor Longuet-Higgins' review, whicin he believes are open to criticism.

Professor Longuet-Higgins now writes: First, my remark that Dr Altmann's groups are "virtually useless for classifying the rotational levels of non-rigid molecules" might have ben misconstrued to imply that the book deals explicitly with rotational levels. This it does not do, nor does it claim to do so, although Dr Altmann does give reference to theoretical work which is relevant to the treatment of rotational levels.

Secondly, the statement in the review that the symmetry operations relevant to an overall molecular state must "commute with the full molecular hamiltonian" could be taken to imply that the book defines symmetry operations to commute only with the Born-Oppenheimer hamiltonian; that is not the case.

Finally, my statement that "the book is, unfortunately, riddled with misprints ... spelling mistakes . could be read as suggesting that such errors were numerous. In fact I found very few.

I apologise unreservedly to $\mathrm{Dr}$ Altmann for having allowed these matters to escape my attention.

H. C. Longuet-Higgins 